\title{
Sequence Analysis of Txk from the Scorpion Mesobuthus eupeus Venom Glands Using Semi-Nested RT-PCR
}

\author{
${ }^{1}$ Ghafar Eskandari, ${ }^{2}$ Abbas Jolodar and ${ }^{3}$ Ahmad Taghavi Moghadam \\ ${ }^{1}$ Department of Agriculture, Islamic Azad University, Izeh Branch, Iran \\ ${ }^{2}$ Department of Basic Sciences, Faculty of Veterinary Medicine, \\ Shahid Chamran University of Ahvaz, Ahvaz, Iran, \\ ${ }^{3}$ Scientific Board of Razi Institute, Ahvaz, Iran
}

\begin{abstract}
Natural toxins are useful probes for evaluating the involvement of $\mathrm{K}+$ channels in cell activity and for investigating $\mathrm{K}+$ channel structure and localization. In recent years, peptide toxins that block various $\mathrm{K}+$ channels with high affinity have been purified from diverse animal venoms. One polypeptide beta neurotoxin named Txk was isolated from the venom of scorpion Mesobuthus eupeus of Khuzestan. This toxin consists of 91 amino acid residues which modulate voltage-gated sodium channels gating. In this study, cDNA of Txk $\beta$-toxin was amplified and sequence of beta neurotoxin compared with $M$. martensii occitanus Israelis and Tityus costatus however, the comparison suggests that the length of the peptide is close to the long-chain potassium ion channel blocker peptide family.
\end{abstract}

Key words: Sequence analysis, K+ channels, beta neurotoxin, scorpion venom, amino acid, Armenia

\section{INTRODUCTION}

Scorpion venom contains a complex mixture of bioactive peptides, non-disulfide-bridge peptides that exhibit potentiating activity, anti-microbial action, hemolytic and immune-modulatory functions among which are peptides responsible for the neurotoxin effects observed during envenomation caused by scorpion stings. A subset of arthropod venoms are complex mixtures of highly evolved peptide libraries with toxin activities that include anti-microbial pore forming and ion channel (Ma et al., 2009). Peptides with high affinity and specificity, targeted to ion-channels were reported to exist in these venoms. Many of these peptides have been widely used for identification, isolation and physiological characterization of ion-channel proteins. The best known are those that recognize $\mathrm{K}+$ and $\mathrm{Na}+$ channels (Elgar et al., 2006; Gandhe et al., 2007) which are usually rich disulfide-containing peptides. Scorpion toxins that modulate voltage-gated sodium channels gating are divided into $\alpha$ and $\beta$ classes according to their mode of action and binding features to distinct receptor sites (Elgar et al., 2006; Wei-Jun and Chao-Qun, 2009). $\beta$-toxins are further classified into depressant and excitatory toxins. $\beta$-toxins shift the voltage dependence of channel activation to more negative membrane potentials upon binding to receptor site 4 , assigned mainly to external loops in domain 2 of mammalian and insect voltage-gated sodium channels (Elgar et al., 2006). These peptides have the potential to combat cancer tumors and a variety of bacterial and fungal infections (Elgar et al., 2006).

At present, the research is mainly focused on the isolation and identification of Txk $\beta$-toxin proteins from scorpion Mesobuthus eupeus from Khuzestan province. Mesobuthus eupeus is one of the most frequent scorpions from Mesobuthus species and is belong to Buthidae family. This scorpion is reported from the most area of Iran, especially Khuzestan (Dehghani et al., 2009). In the present study, cDNA of Txk $\beta$-toxin was amplified and characterized from scorpion Mesobuthus eupeus from Khuzestan province.

\section{MATERIALS AND METHODS}

Scorpion samples: Scorpion Buthida Mesobuthus eupeus Khuzestan species were collected from Khuzestan province (Iran) and transported to the laboratory reference of the Razi Institute where they were killed 2 days after manual extraction of their venom to allow the toxin-producing cells of the venom glands to enter the secretory phase. Twenty separated venom glands are used for total RNA extraction.

Corresponding Author: Ghafar Eskandari,Department of Agriculture, Islamic Azad University, Izeh Branch, Iran 
Total RNA extraction: Total RNA was extracted from the venom glands of scorpions using RNA ${ }^{\mathrm{TM}}$ (Cinagene, Iran) according to the manufacture procedure. RNA pellet was dissolved in DEPC-ddH $\mathrm{H}_{2} \mathrm{O}$ and used for cDNA synthesis immediately.

cDNA library synthesis: Thev cDNA was synthesized with the extracted total RNA as template and $\operatorname{modT}$ (modified oligodT) (5-cgcggatccatg caaaggaatctggtcgt - $3^{\prime}$ ) as primer. ModT was added to extracted RNA and incubated in $70^{\circ} \mathrm{C}$ for $5 \mathrm{~min}$ and then immediately on ice for $2 \mathrm{~min}$. Then, $5 \mathrm{X}$ buffer, dNTP, Ribolock, Reverce transcriptase enzyme and $\mathrm{ddH}_{2} \mathrm{O}$ were added to samples and incubated for $60 \mathrm{~min}$ in $42^{\circ} \mathrm{C}$. Samples were incubated $10 \mathrm{~min}$ in $70^{\circ} \mathrm{C}$ and immediately on ice.

Semi-nested RT-PCR amplification: Amplification of Txk cDNA was performed using Semi-nested RT-PCR strategy. The 1st round of PCR was performed using modT-R ( $5^{\prime}$-cccagatctcgagctcagtg- $\left.3^{\prime}\right)$, Txk-F ( $5^{\prime}$-gcgcgga tccaagatggcttcaagtttcatt- $3^{\prime}$ ) primers and synthesized cDNA as template. Second round of PCR was performed using Txk-F and Txk-R (5'-gcgcaagctttacagttgttatca ttgataaattg-3') primers. PCR products of initial amplification were used as template for the 2 nd round of amplification. The PCR conditions for both rounds were 35 cycles with denaturation at $94^{\circ} \mathrm{C}(40 \mathrm{sec})$, anealing at $56^{\circ} \mathrm{C}(90 \mathrm{sec})$ and extention at $72^{\circ} \mathrm{C}(1 \mathrm{~min})$ with a initial denaturation at $95^{\circ} \mathrm{C}(5 \mathrm{~min})$ and final extention at $72^{\circ} \mathrm{C}(10 \mathrm{~min})$. Amplification products were separated by $1 \%$ agarose gel electrophoresis and visualized by UV transilluminator.

DNA sequencing: The amplified cDNA fragments were purified from agarose gel by QIAquick agarose gel extraction kit (www.fermentas.com) and then sent to Kawsar Biotech Company for nucleotide sequencing.

Sequence analysis: Sequence was compared with GenBank database using the BLAST software from NCBI site (http://www.ncbi.nlm.nih.gov). The tool software available at the Expasy website (http://ca.expasy.org/ tools/pi_tool.html) was used to convert nucleotide sequence to amino acid. The molecular weight and isoelectric point was estimated using ProtParam tool (http://www.expasy.org/tools/protparam.html). The signal peptide was predicted by SignalP (http://www.cbs.dtu.dk/ services/SignalP/). Multiple sequence alignments were done using the CLUSTAL_W program and edited with the BOXSHADE software (http://www.ch.embnet.org/ software/BOX_form.html). The SBASE online software (http:/hydra.icgeb.trieste.it/sbase/) was used to determine the conserved domains.

\section{RESULTS AND DISCUSSION}

Characterizationand semi-nested RT-PCRamplification of the Txk $\beta$-toxin gene cDNA: In this research, we starting with $4 \mu \mathrm{g}$ of total RNA from $0.5 \mathrm{~g}$ of tissues were obtained using $\mathrm{RNX}^{\mathrm{TM}}$ regent according to the standard protocol. To characterize and assay the mRNAs, single strand cDNAs were synthesized and electrophoresis on a $1 \%$ agarose gel cDNA fragments of about $273 \mathrm{bp}$ encoding a beta neurotoxin from $M$. mupeus were amplified using RT-PCR technique. The size of the coding region was $273 \mathrm{bp}$. We used PCR strategy to obtain a cDNA probe for the screening of the cDNA library (Fig. 1).

M-Marker: A putative 19-amino-acids length signal peptide was identified and glycine at the position 20 was assumed to represent the start of the mature protein. According to the sequencing results, the peptide coding sequence was $273 \mathrm{bp}$ in length, encoding for 91 aa residue peptide with molecular weight $10.207 \mathrm{kDa}$ and theoretical isoelectric point of $9 / 06$.

The cDNA homology search against the GenBank database revealed that the amino acid sequence of beta neurotoxin Txk shared high sequence similarity with beta neurotoxins of other scorpions. Particularly, the deduced amino acid sequence exhibited 96,94 and $67 \%$ similarities with the homologous Long-chain potassium ion channel blocker from M. martensii, Buthus occitanus Israelis and Tityus costatus, respectively.

In this study, beta-neurotoxin Txk from venom glands of $M$. eupeus scorpion from Khuzestan province was identified. The amino acid sequence of beta-neurotoxin Txk was compared to beta neurotoxins from several scorpions which revealed higher homology within these species. M. martensii beta-neurotoxin Txk has

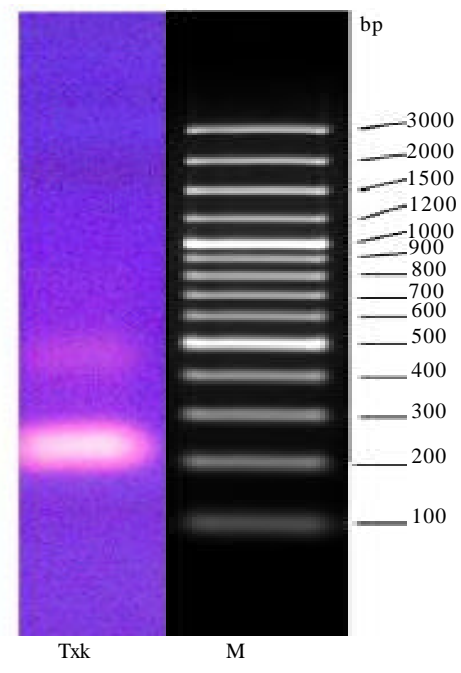

Fig. 1: PCR amplification of beta neurotoxin Txk cDNA 
demonstrated the highest homology with beta-neurotoxin Txk determined in this study whereas, the least similarity was found with Tityus costatus beta-neurotoxin and shows homology to the published leader peptides of $\mathrm{KTx}$, Ktx2 (Legros et al., 1996) and Tsk (Wei-Jun and Chao-Qun, 2009), $\mathrm{K}+$ channel blockers from scorpion venoms.

\section{CONCLUSION}

According to the differences between $M$. martensii and Tityus costatus, it is concluded that $M$. eupeus of Khuzestan and Tityus costatus, $M$. eupeus belong to the different subspecies. The beta neurotoxin from $M$. eupeus belongs to the Toxin_3 superfamily. The length of the peptide is close to the long-chain potassium ion channel blocker peptide family. The largest group of $\mathrm{K}+$ channel peptide inhibitors is the family of neurotoxic peptides found in scorpion venoms. These peptides block in nanomolar concentrations both voltagegated and $\mathrm{Ca}^{2+}$ activated $\mathrm{K}+$ channels in a wide variety of cell types and generally contain 3,140 amino acid residues crosslinked by three or four disulfide bridges (Whetstone and Hammock, 2007; Ma et al., 2009).

\section{RECOMMENDATION}

The comparison suggests that the length of the peptide is close to the Long-chain potassium ion channel blocker peptide family.

\section{ACKNOWLEDGEMENTS}

This research was supported by the Razi Reference Laboratory of Scorpion Research (RRLS), Ahvaz, Iran. The researchers are grateful to Dr. Ghaemmaghami, Mr. Taghavi, Mr. Masihipour and Mr. Bahrain for their kind support.

\section{REFERENCES}

Dehghani, R., N.D. Djadid, D. Shahbazzadeh and S. Bigdelli, 2009. Introducing Compsobuthus matthiesseni (Birula, 1905) scorpion as one of the major stinging scorpions in Khuzestan, Iran. Toxicon, 54: $272-275$.

Elgar, D., J.D. Plessis and L.D. Plessis, 2006. Cysteine-free peptides in scorpion venom: Geographical distribution, structure-functionrelationship and mode of action. Afr. J. Biotechnol., 5: 2495-2502.

Gandhe, A.S., G. Janardhan and J. Nagaraju, 2007. Immune upregulation of novel anti-bacterial proteins from silkmoths (Lepidoptera) that resemble lysozymes but lack muramidase activity. Insect Biochem. Mol. Biol., 37: 655-666.

Legros, C., R. Oughuideni, H. Darbon, H. Rochat, P.E. Bougis and M.F. Martin-Eauclaire, 1996. Characterization of a new peptide from Tityus serrulatus scorpion venom which is a ligand of the apamin-binding site. FEBS Lett., 390: 81-84.

Ma, Y., R. Zhao, Y. He, S. Li and J. Liu et al., 2009. Transcriptome analysis of the venom gland of the scorpion Scorpiops jendeki: Implication for the evolution of the scorpion venom arsenal. BMC Genomics, 10: 290-290.

Wei-Jun, M. and H. Chao-Qun, 2009. Molecular cloning, characterization, expression and anti-bacterial analysis of a lysozyme homologue from Fenneropenaeus merguiensis. Mol. Biol. Rep., 36: 1587-1595.

Whetstone, P.A. and B.D. Hammock, 2007. Delivery methods for peptide and protein toxins in insect control. Toxicon, 49: 576-596. 